\title{
NOUVELLE
}

\section{Les lésions de}

I'endothélium vasculaire

\section{Un rôle clef dans les atteintes multiviscérales de la COVID-19?}

Annabelle Dupont, Mickaël Rosa, Antoine Rauch, Sophie Susen
Univ. Lille, Inserm, CHU Lille,

Institut Pasteur de Lille,

U1011- EGID (European Genomic Institute for Diabetes),

Boulevard du Pr Leclerc, 59037 Lille, France.

annabelle.dupont@univ-lille.fr
> En décembre 2019, le SARS-CoV-2 (severe acute respiratory syndromecoronavirus-2), responsable de la COVID-19 (coronavirus disease-2019), a émergé en Asie et s'est propagé dans le monde. À ce jour, plus de 380 millions de personnes dans le monde ont été infectées, et plus de 5,7 millions de décès ont été enregistrés par l'Organisation mondiale de la santé. Dans la majorité des cas, la COVID-19 est une infection bénigne limitée aux voies aériennes supérieures. Toutefois, 10 à $15 \%$ des patients infectés développent une pneumopathie qui peut évoluer vers un syndrome de détresse respiratoire aiguë. De nombreuses études cliniques rapportent également une incidence particulièrement élevée d'évènements thrombotiques veineux et artériels chez les patients hospitalisés pour une infection par le SARS-CoV-2 [1], et les complications extra-pulmonaires sont fréquentes (insuffisance rénale, cytolyse hépatique, myocardite, etc.) [2]. D’ailleurs, contrairement à d'autres infections respiratoires virales apparentées, les analyses histopathologiques post-mortem ont montré la présence de lésions endothéliales diffuses et de thromboses de la microcirculation dans les poumons, les reins, la peau, le foie ou le système digestif [3]. Ces microthrombus ont par ailleurs la caractéristique d'être particulièrement riches en fibrine et en NET (neutrophil extracellular traps), des pièges extracellulaires produits par les granulocytes neutrophiles [4]. Ces complexes extracellulaires, constitués principalement de filaments d'ADN, d'histones et de protéines granulaires, sont libérés par les granulocytes neutrophiles lorsqu'ils sont activés. Ils jouent un rôle majeur dans les processus d'immunothrombose, un terme proposé en 2013 par Engelmann et Massberg, et qui désigne les multiples interactions entre l'immunité innée et l'hémostase, conduisant à la formation de thrombus, notamment dans la microcirculation [5].

Les cellules endothéliales, qui tapissent la surface interne de la paroi des vaisseaux sanguins, jouent un rôle capital dans l'homéostasie vasculaire en contrôlant la perméabilité vasculaire, la vasomotricité et les réactions inflammatoire et immunitaire, et en inhibant la coagulation. Lorsqu'elles sont lésées, ces cellules perdent le contrôle de ces fonctions. Elles libèrent alors dans la circulation de nombreuses molécules capables de moduler l'agrégation des plaquettes, la coagulation ou la fibrinolyse (dont le facteur de von Willebrand, l'inhibiteur de l'activateur du plasminogène-1, l'inhibiteur de la voie du facteur tissulaire, la thrombomoduline soluble, le syndécane-1, etc.), et acquièrent un phénotype prothrombotique, proinflammatoire et proadhésif pour les leucocytes, les NET et les plaquettes.

Ce dysfonctionnement endothélial diffus peut être la conséquence d'un effet cytopathogène direct du SARS-CoV-2 sur les cellules endothéliales, qui présentent à leur surface deux protéines indispensables à l'entrée du virus: I'enzyme de conversion de l'angiotensine
ACE2 (angiotensin-converting enzyme 2) et la protéase transmembranaire à sérine TMPRSS2 (transmembrane serine protease 2). L'observation, en microscopie électronique, de tissus de patients décédés de la COVID-19 a d'ailleurs montré que des particules virales sont présentes dans les cellules endothéliales lésées ou apoptotiques [6]. Cependant, la preuve d'une réplication active du virus dans les cellules endothéliales humaines n'a pas encore été apportée, et les signes d'infection de ces cellules sont rarement observés à un stade avancé de la maladie.

Les résultats de nos travaux ont apporté un éclairage nouveau sur les mécanismes participant à l'infection de ces cellules [7]. En effet, nous avons observé que le plasma de patients atteints d'une forme sévère de la COVID-19, lorsqu'il est prélevé à la phase aiguë de l'infection, avait, in vitro, un effet cytotoxique sur les cellules endothéliales de la microcirculation pulmonaire. Cet effet est observé après seulement une heure de contact du plasma de patients avec les cellules en culture, ce qui exclut un effet cytopathogène direct du SARS-CoV-2. Ainsi, dans les formes sévères de la COVID-19, à l'action directe du SARS-CoV-2 sur les cellules endothéliales, s'ajoutent d'autres mécanismes indirects, probablement multifactoriels, qui participent à l'altération de l'endothélium. II convient de noter que la cytotoxicité induite par le plasma de ces patients est positivement corrélée non seulement à leurs taux circulants de marqueurs de l'atteinte endothéliale et du dérèglement de la réponse immu- 


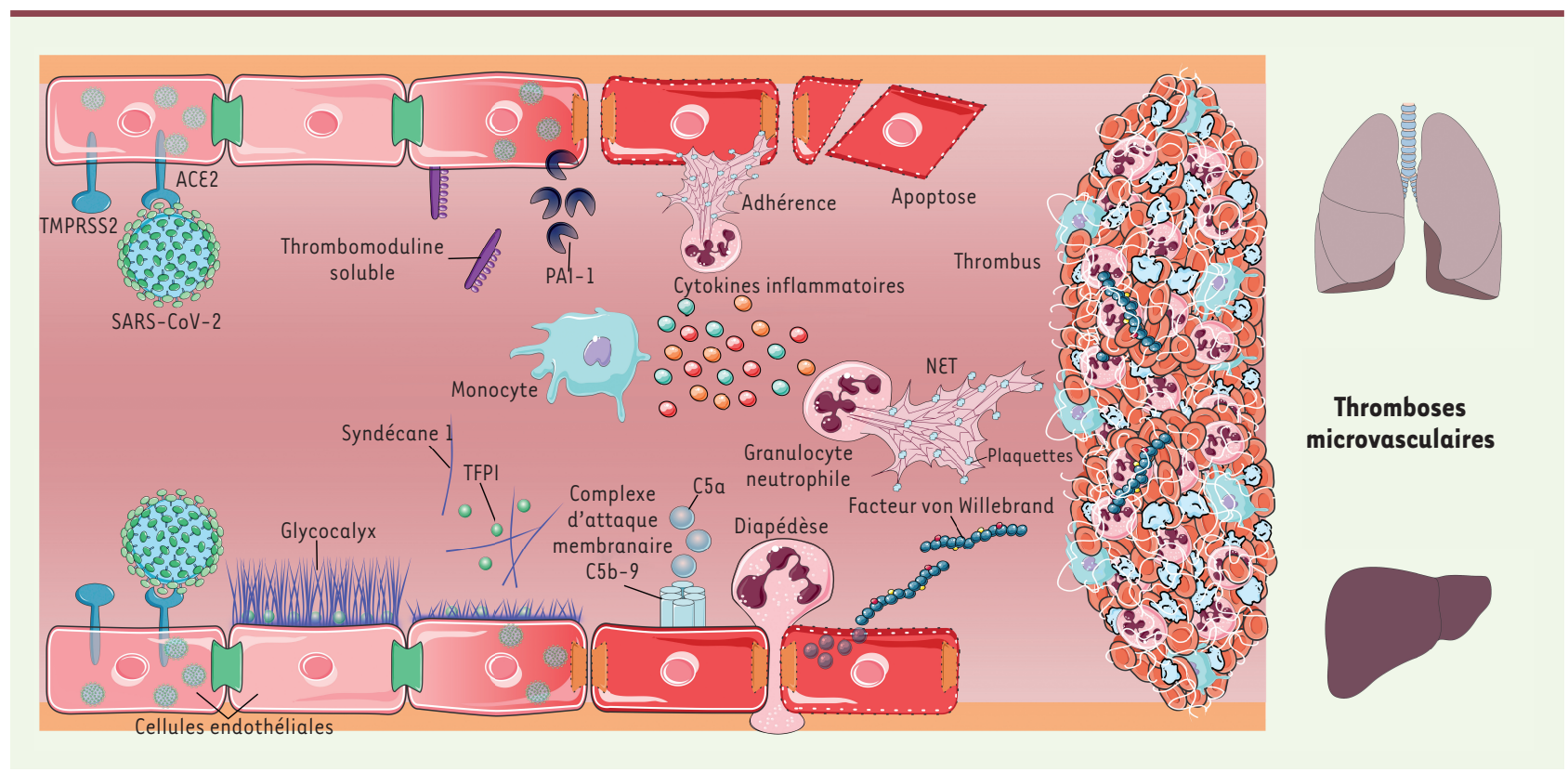

Figure 1. Rôle clé du dysfonctionnement de l'endothélium et de l'immunothrombose dans la pathogenèse des microthrombus et des défaillances d'organes dans les cas d'infection sévère par le virus SARS-CoV-2. ACE2 : angiotensin-converting enzyme 2 ; NET : neutrophil extracellular trap ; SARS-CoV-2 : severe acute respiratory syndrome-coronavirus-2; PAI-1 : plasminogen activator inhibitor 1 ; TFPI : tissue factor pathway inhibitor ; TMPRSS2 : transmembrane protease serine 2 ; C5a : composant 5 a du complément.

nitaire, mais également à la sévérité de leurs atteintes tissulaires. Ainsi, le dysfonctionnement endothélial diffus, caractéristique des formes sévères de la COVID-19, jouerait donc, en lien avec les processus immunitaires, un rôle majeur dans la survenue des thromboses de la microcirculation à l'origine des complications pulmonaires et extra-pulmonaires de la maladie.

Pour explorer cette hypothèse, nous avons entrepris l'étude d'un groupe de 82 patients atteints de COVID-19 et admis en unité de soins intensifs au centre hospitalo-universitaire de Lille, en mesurant, à l'admission des patients, les taux circulants de plusieurs biomarqueurs du dysfonctionnement endothélial (facteur de von Willebrand, inhibiteur de l'activateur du plasminogène-1, inhibiteur de la voie du facteur tissulaire, thrombomoduline soluble et syndécane) et de la réponse immunitaire: cytokines proinflammatoires (interleukine 6 et TNF- $\alpha$ [tumor necrosis factor- $\alpha]$ ), sous-unité $\alpha$ du récepteur de l'interleukine 2, compo- sants des NET (ADN libre, nucléosomes, complexes myéloperoxydase-ADN) et marqueurs d'activation du complément (fragments $\mathrm{C} 5 \mathrm{a}$ et complexe d'attaque membranaire, (5b-9) [8]. Nous avons également analysé la composition de thrombus collectés chez des patients vivant sous oxygénation extracorporelle de membrane, un dispositif d'assistance respiratoire ou cardiaque utilisé en dernier recours dans les unités de soins intensifs. Parmi les 82 patients inclus dans l'étude, $73 \%$ ont présenté une défaillance respiratoire à leur admission ou au cours des 14 jours suivants, $38 \%$ ont présenté une défaillance hépatique, et $60 \%$ une défaillance multiviscérale, et, 28 jours après leur admission, $27 \%$ des patients inclus dans l'étude sont décédés.

Nous avons tout d'abord observé que les taux élevés des marqueurs du dysfonctionnement endothélial et des NET étaient associés à la survenue de défaillances respiratoires, hépatiques ou multiviscérales, et de décès, indépendamment de l'âge, du sexe et de l'indice de masse corporelle des patients. Ces résultats renforcent l'hypothèse d'un rôle direct du dysfonctionnement endothélial et des NET dans les défaillances d'organes des formes sévères de la COVID-19. De plus, l'existence d'une corrélation positive entre la sévérité de l'atteinte de l'endothélium et les taux circulants de cytokines et de marqueurs d'activation du complément et des NET a révélé une interaction étroite entre les processus inflammatoires et les lésions endothéliales conduisant aux défaillances d'organes. Enfin, nous avons observé que les thrombus, extraits des circuits d'oxygénation extracorporelle de membrane utilisés chez huit patients vivants et atteints d'une forme sévère de la COVID-19, étaient très riches à la fois en cellules, principalement des granulocytes neutrophiles, et en NET, ainsi qu'en facteur de von Willebrand, par rapport à des thrombus de patients témoins. Le facteur de von Willebrand libéré par les cellules endothéliales endommagées pourrait ainsi former, avec les NET, des complexes favorisant 
l'adhérence des plaquettes et la formation des thrombus, comme cela a été montré in vitro et dans différents modèles animaux d'immunothrombose dans d'autres contextes que l'infection par le SARS-CoV-2.

$\varepsilon$ n conclusion, ces résultats fournissent des preuves supplémentaires que le dysfonctionnement endothélial et la réponse immunitaire, notamment l'activation des granulocytes neutrophiles, jouent un rôle majeur dans la pathogenèse des microthrombus et des défaillances d'organes dans les cas d'infection sévère par le SARS-CoV-2. Ces mécanismes, complexes et interdépendants, entretiennent des boucles d'amplification, facilitant la propagation des dommages de l'endothélium, le dérèglement du système immunitaire, et les processus thrombotiques (Figure 1). Cibler ces processus en agissant notamment sur le facteur de von Willebrand ou la formation des NET pourrait ainsi représenter une stratégie thérapeutique efficace pour prévenir la formation des thrombus dans la microcirculation et améliorer le pronostic de la maladie. Certaines molécules thérapeutiques sont d'ailleurs déjà à l'étude dans ce contexte. C'est le cas, par exemple, de la $\mathrm{N}$-acétylcystéine, qui réduit l'activité du facteur de von Willebrand, ou de la DNase I humaine recombinante, une enzyme qui dégrade les NET: pour ces deux molécules, les premiers résultats d'essais cliniques dans la COVID-19 semblent prometteurs $[9,10]$.

Ces travaux apportent ainsi des éléments majeurs pour comprendre les mécanismes impliqués dans la pathogenèse des complications pulmonaires, hépatiques et multiviscérales survenant fréquemment chez les patients atteints de la COVID-19 et nécessitant une hospitalisation en unité de soins intensifs. Notre compréhension de ces mécanismes doit encore progresser, un préalable indispensable pour identifier ensuite des biomarqueurs pertinents pour dépister les patients les plus à risque de développer des formes sévères de la COVID-19 et proposer des interventions thérapeutiques efficaces pour contrôler ces formes graves. $\diamond$

Vascular endothelial damages: A key role in COVID-19 multiorgan failure?

\section{LIENS D'INTÉRÊT}

Les auteurs déclarent n'avoir aucun lien d'intérêt concernant les données publiées dans cet article.

\section{RÉFÉRENCES}

1. Castro RA, Frishman WH. Thrombotic complications of COVID-19 infection: A review. Cardiol Rev $2021 ; 29$ : 43-7.

2. Gupta A, Madhavan MV, Sehgal K, et al. Extrapulmonary manifestations of COVID-19. Nat Med $2020 ; 26: 1017-32$.

3. Ackermann M, Verleden SE, Kuehnel M, et al. Pulmonary vascular endothelialitis, thrombosis, and angiogenesis in Covid-19. N Engl J Med 2020 ; 383 : 120-8.

4. Leppkes M, Knopf J, Naschberger $\varepsilon$, et al. Vascular occlusion by neutrophil extracellular traps in COVID19. EBioMedicine $2020 ; 58: 102925$.

5. Engelmann B, Massberg S. Thrombosis as an intravascular effector of innate immunity. Nat Rev Immunol 2013; 13 : 34-45.

6. Varga Z, Flammer AJ, Steiger P, et al. Endothelial cell infection and endotheliitis in COVID-19. Lancet 2020 ; 395 : 1417-8.

7. Rauch A, Dupont A, Goutay J, et al. Endotheliopathy is induced by plasma from critically ill patients and associated with organ failure in severe COVID-19. Circulation 2020 ; 142 : 1881-4.

8. Dupont A, Rauch A, Staessens S, et al. Vascular endothelial damage in the pathogenesis of organ injury in severe COVID-19. Arterioscler Thromb Vasc Biol 2021; 41 : 1760-73.

9. Assimakopoulos SF, Aretha D, Komninos D, et al. $\mathrm{N}$-acetyl-cysteine reduces the risk for mechanical ventilation and mortality in patients with COVID-19 pneumonia: a two-center retrospective cohort study. Infect Dis (Lond) 2021 ; $53:$ 847-54.

10. Fisher J, Mohanty T, Karlsson CAQ, et al. Proteome profiling of recombinant DNase therapy in reducing NETs and aiding recovery in COVID-19 patients. Mol Cell Proteomics $2021 ; 20: 100113$.

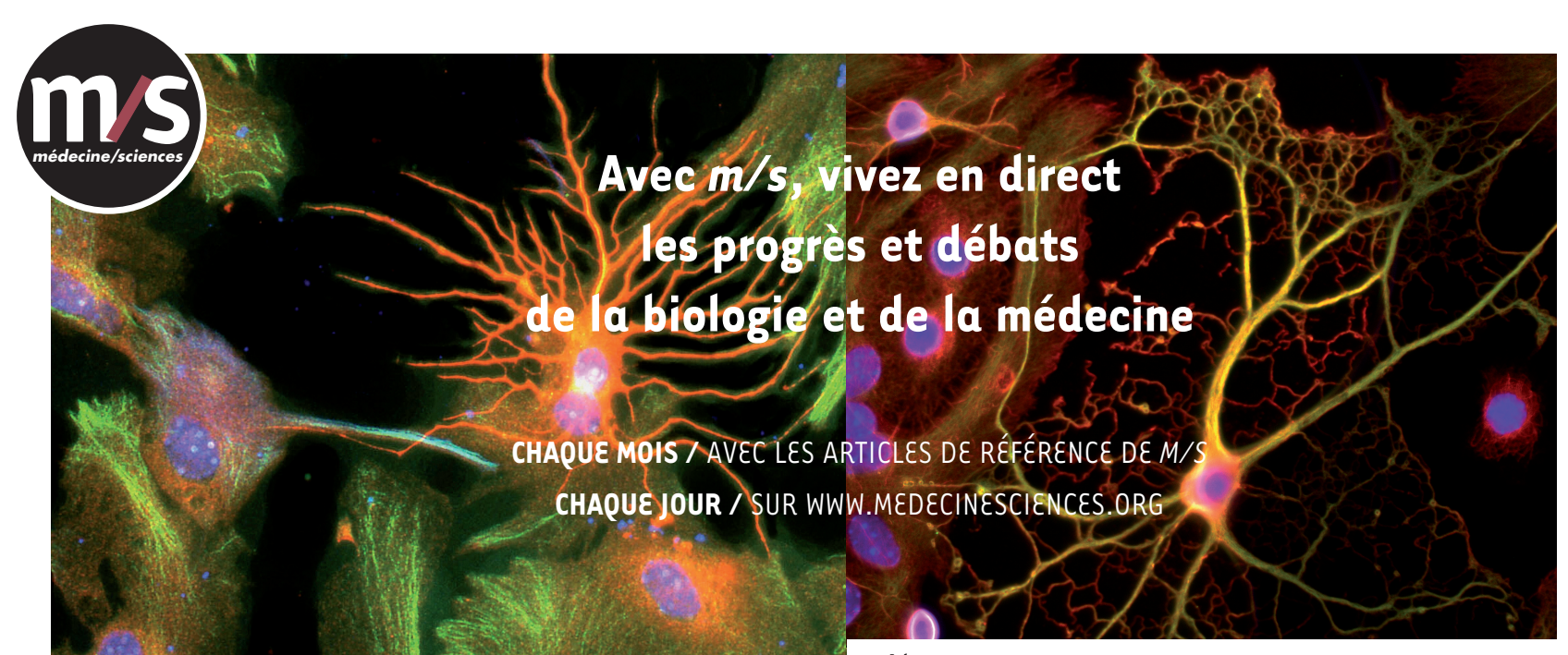

Abonnez-vous sur

www.medecinesciences.org 